\title{
LE MARCHÉ DU POISSON D'EAU DOUCE ${ }^{(1)}$
}

\author{
par M. DE LA SELLE \\ Pisciculteur a Bière (Loir-et-Cher)) \\ Président de l'Union? Nationale des Syndicats de l'étang.
}

Je ne désire pas ici aborder un sujet technique, mais seulement entretenir les lecteurs du Bulletin français de pisciculture d'une question économique et plus prosaïque, celle du marché du poisson d'eau douce et même, plus spécialement, de la commercialisation du poisson d'étangs.

Cette question est, je dirai, très à l'ordre du jour, car la vente du poisson d'eau douce est devenue difficile; il y a crise. Cette crise peut-elle être conjurée? Les producteurs peuvent-ils avoir l'espoir de vendre le poisson tel qu'il est produit actuellement ou doivent-ils, au contraire, modifier leur production? La question mérite d'être étudiée. Je dois dire, quant à moi, mais tous ne sont pas de mon avis, que la production devra être modifiée et se plier aux exigences de la clientèle.

Pour bien fixer les idées, je voudrais revenir un peu en arrière et examiner ce qu'était la production il y a d'assez longues années, ce qu'elle était il y a très peu d'années ; enfin, ce qu'elle est actuellement et quelles sont les tendances de l'avenir.

Je crois que, depuis mettons 1500 jusque vers 1900 , la production des étangs n'a pas varié. On produisait des Carpes, des Brochets, de la blanchaille, un peu au petit bonheur; on pechait tous les $2,3,4$ ans, on remettait dans l'étang ce qui n'était pas marchand et... on ne s'occupait plus de rien.

J'ai retrouvé dans les archives de ma famille certains chiffres assez intéressants; ainsi, dans un étang de 50 hectares que je peux suivre de très près, je vois que mon grand-père prenait tous les 3,4 ou 5 ans, de 3.500 à 4.000 kilos de poissons variés. La pêche était solennelle, le marchand arrivait avec une douzaine de voitures chargées de tonneaux pleins d'eau qui, parfois, faisaient deux et même trois voyages. Le poisson se vendait facilement à un prix équivalent à celui du kilo de porc ou de la livre de beurre, c'est-à-dire de $0 \mathrm{fr}$. 80 à 1 franc le kilo de Carpe, $1 \mathrm{fr} .25$ le kilo de Brochet, 1 fr. 50 le kilo d'Anguille.

Il est vrai qu'à cette époque, la marée n'arrivait pas dans les campagnes et que les habitants ne pouvaient manger - en fait de poisson que le hareng saur ou le poisson d'étang.

(1) Résumé d'une communication faite à la Société centrale d'Aquiculture et de Pêche, le 26 avril 1951. 
Ce n'est que vers 1905-1910 que M. de Neufbourg et aussi, je crois, M. Hirsch, ont commencé à importer des Carpes de Silésie. En même temps que les Carpes, ces producteurs importaient les méthodes silésiennes d'élevage. M. de Neufbourg, en particulier, faisait avec ardeur une campagne pour la pêche à un an et, donnant l'exemple, prouvait qu'en six mois on pouvait faire une excellente Carpe de 3 livres.

Je veux ici rendre un très spécial hommage à $M$. de Neufbourg, qui a révolutionné la culture des étangs et dont la "Carpe Royale " a fait faire des progrès considérables à notre production. Il n'y a plus aucun rapport entre les pêches actuelles de plusieurs tonnes de Carpes toutes de la même taille avec un indice oscillant entre 2, 3 et 2,6 et les pêches d'autrefois où l'on trouvait un Brochet de 27 livres avec de pauvres " peinards ".

Mais, dès après la guerre de 1914, les difficultés de vente de la Carpe ont commencé ; $\mathbf{M}$. de Neufbourg a inauguré alors une certaine publicité, par tracts et brochures extrêmement bien faits et il a pu exporter en Allemagne. Malgré tout, la Carpe boudait déjà ; il est vrai que la production, avec les nouvelles méthodes, avait beaucoup augmenté. Les producteurs, devant la carence des marcahands et des intermédiaires, organisaient des ventes au détail. Bref, les difficultés commençaient à se manifester.

Pour les carpiculteurs, la guerre de 1939-1945, avec toutes ses restrictions, a été une aubaine : pas de viande, pas de poisson de mer, le public se décidait à manger de la Carpe et trouvait même que ce n'était pas « si mauvais que cela». Les prix taxés, par contre, étaient médiocres.

Nous avons bien essayé alors d'introduire la Carpe dans le public sous des formes variées et, en particulier, fumée ; mais ces efforts, couronnés de succès pendant quelques années, sont actuellement devenus stériles. Les difficultés de vente n'ont fait qu'augmenter et le marasme est revenu, plus grand que jamais.

Or, la Carpe actuelle est incontestablement bien meilleure que celle produite autrefois; tous les gens à qui j'ai fait manger de ce poisson m'ont dit : c'est excellent! Je ne pense donc pas, pour ma part, que la mévente vienne du produit lui-même, dont le prix est vraiment très abordable si on le compare à celui de la viande et dont la qualité est certaine.

La marée, me direz-vous, se vend mieux. C'est exact; mais étudions, si vous le voulez bien, les prix de cette marée. Vous constaterez qu'en 1951 le Colin vaut deux fois et même trois fois le prix de la Barbue ; or il n'y a pas de comparaison, à mon sens, du point de vue gastronomique, entre ces deux poissons. Du reste, il y a cinquante ans, la Barbue valait plus cher que le Colin. La raison de cet écart réside, selon moi, dans le fait que le Colin se coupe en tranches, en darnes, qu'en dix minutes ces darnes sont cuites dans une poêle, tandis que, pour cuire une Barbue, il faut une turbotière, un court-bouillon, un fourneau, une cuisinière.

Or, il n'y a plus maintenant de turbotières, de fourneaux, ni surtout de cuisinières.

Les femmes, je n'ose plus dire les ménagères, ne veulent plus faire de 
cuisine : vite le gaz, le beefsteak, à la rigueur les nouilles ou, plutôt, vite la tranche de jambon ou de pâté. Adieu pot-au-feu, ragoûts, et aussi Carpes au four! Car la Carpe n'est bonne que grosse et cuisinée, c'est-àdire vidée, garnie, etc. Voilà pourquoi, je crois, on ne mange plus de Carpes.

Je ne pense pas que, même avec une publicité intense et bien faite (que nous, carpiculteurs - et ce, même avec l'aide des Eaux et Forêts nous sommes bien incapables de financer), on pourrait remonter ce courant; il est entré dans les mœurs, il faut en prendre son parti. Le drame de la mévente de la Carpe est le même que celui du Pot-au-feu.

Faut-il donc renoncer à l'exploitation des étangs dont la Carpe représente $85 \%$ de la production? Je ne le pense pas.

Je constate, en effet, que la pêche sportive, ou simplement même je dirais : " de repos ", se développe de plus en plus; les sociétés sont nombreuses et riches. Les pêcheurs augmentent sur le bord des rivières, ils voudraient prendre du poisson, mais il y a peu de poisson.

Prenons donc contact avec les sociétés de péche et nous, exploitants d'étangs, élevons des poissons que les pêcheurs prendront dans les rivières, et le poisson alors sera mangé. Car le mari décidera sa femme à cuisiner sa pêche, comme sa chasse! Et, en cas de refus, il fera lui même sa cuisine.

La production des étangs devra, évidemment, être modifiée, mais peut-être d'une façon moins grande qu'on peut le penser; car pourquoi ne ferions-nous pas comme les Belges, et ne pourrions-nous pas habituer les sociétés de pêche à organiser des concours en faisant pêcher des grosses Carpes achetées quelques jours plus tôt ?

Il y a là beaucoup à faire et je serais heureux d'avoir sur ce point l'accord de mes lecteurs, et, s'ils ne sont pas d'accord, d'ouvrir avec eux une courtoise discussion.

Je voudrais terminer sur une note optimiste : en effet, si, grâce aux sociétés de pêche, grâce à quelques exportations, le marché se trouvait dégagé de quelques centaines de tonnes, ce qui n'est pas énorme, l'exploitation des étangs pourrait redevenir très prospère et cela me semble très possible. 